\title{
Sensibilidad a la aspirina y posibilidades de desensibilización
}

\section{Objetivo}

Proveer una estrategia para evaluar y tratar pacientes con sensibilidad a la aspirina, con especial consideración para aquellos con enfermedad coronaria.

\section{Fuente y selección de datos}

Búsqueda en las bases de datos MEDLINE y COCHRANE (19662004) utilizando los términos "aspirin allergy", "coronary artery disease", "aspirin desensitization", "aspirin sensitivity" y revisión de los resúmenes de los congresos americanos de Alergia y Cardiología.

\section{Extracción de datos}

Se incluyeron estudios controlados y de relevancia clínica publicados en inglés. La calidad de los datos fue determinada según su relevancia en el cuidado de los pacientes y su publicación en revistas con comité revisor. Debido a que no existen trabajos aleatorizados en relación con la sensibilidad a la aspirina o los antiinflamatorios no esteroideos (AINE) se incorporaron trabajos de series de casos y guías de práctica clínica de sociedades médicas.

\section{Resultados principales}

La prevalencia en la población general de enfermedad respiratoria (rinitis, asma) inducida por aspirina es aproximadamente del $10 \%$ y a su vez, la de urticaria inducida por aspirina varía del 0,07 al 0,2\%.
El mecanismo más frecuente es la inhibición de la ciclooxigenasa 1 (COX1). Por otro lado, la producción de IgE específica contra los AINE determina algunos casos de urticaria, angioedema y excepcionalmente, anafilaxia..

La mayoría de los pacientes con sensibilidad a la aspirina son pasibles de realizar el tratamiento de desensibilización de manera segura, excepto en los casos de urticaria crónica en los que la reexposición programada al ácido acetilsalicílico u otro AINE suele desencadenar una exacerbación de la sintomatología de hipersensibilidad. Sin embargo, no hay estudios aleatorizados que hayan demostrado específicamente la eficacia de la desensibilización a la aspirina. Además, la experiencia de la desensibilización con aspirina en los pacientes con enfermedad coronaria es muy limitada. Por otra parte, luego de una desensibilización exitosa, la terapia con ácido acetilsalicílico debe ser mantenida indefinidamente para continuar con la prevención de la resensibilización.

\section{Conclusiones}

La sensibilidad a la aspirina es frecuente y la desensibilización puede ser efectuada de manera aparentemente segura en muchos pacientes. Sin embargo, se necesitan más trabajos metodológicamente adecuados para poder determinar la seguridad y eficacia de la desensibilización con ácido acetilsalicílico en pacientes con enfermedad coronaria concomitante ya que las referencias encontradas se hallan limitadas a series de casos.

Fuente de financiamiento: no referida.

\section{Comentario}

La aspirina ocupa el segundo lugar (después de la penicilina) como causa de reacciones adversas por drogas. Dichas reacciones son en su mayoría no alérgicas. Si bien ha sido descripta la reacción mediada por lgE (verdaderamente alérgica) la hipótesis más atractiva acerca de la patogenia de estas reacciones sigue apuntando hacia el disbalance entre los metabolitos del ácido araquidónico $0^{2,3}$ (inhibición de la COX1). El diagnóstico suele hacerse por el interrogatorio y la conducta recomendada en primer lugar sigue siendo la evitación del fármaco. Sólo en situaciones en las que no se puede prescindir del mismo y en ausencia de un fármaco alternativo, se aconsejaría realizar desensibilización, procedimiento que si bien es llevado a cabo con frecuente éxito y sin complicaciones en la mayoría de los casos, no está exento de riesgos.

Distintos protocolos han sido diseñados a tal efecto; cualquiera sea el que se lleve adelante debería realizarse en sitios con personal entrenado y con acceso inmediato a unidades de cuidados intensivos.

\section{Conclusión de la comentadora}

En casos excepcionales podría intentarse la desensibilización a la aspirina, procedimiento no excento de riesgos y que todavía requiere de mayores investigaciones para avalar su seguridad.

Roxana Haas [ Sección Alergia del Servicio de Clínica Médica. Hospital Italiano de Buenos Aires. ]

Haas R. Sensibilidad a la aspirina y posibilidades de desensibilización. Evid. práct. ambul. 9(4) ;101 Jul-Ag. 2006. Comentado de: Raghava R. Gollapudi, MD; Paul S. Teirstein, MD; Donald D. Stevenson, MD; Ronald A. Simon, MD. Aspirin Sensitivity. Implications for Patients With Coronary Artery Disease. JAMA. 2004;292:3017-3023. PMID: 15613671.

\section{Referencias}

1. Chou E, Minutello R, Parikh M, Bergman G, Chiu Wong S, Hong MK. Can we identify vulnerable patients at risk for ST-segment elevation myocardial infarction based on their clinical characteristics? Coron Artery Dis. 2004. 15 :467-469.

2. Parmet S, Lynm C, Glass RM. JAMA patient page. Aspirin Sensitivity. JAMA 2004. 292 : 3098.

3. Greenberger J, Bingham C 3rd, Abramson S, Reed G, Sebaldt R, Kremer J. Effect of cardiovascular comorbidities and concomitant aspirin use on selection of cyclooxygenase inhibitor among rheumatologists. Arthritis Rheum. 2005. 53: 12-17.

4. Schleinitz M, Heidenreich P. A cost effectiveness analysis of combination antiplatelet therapy for high risk acute coronary syndromes: clopidogrel plus aspirin versus aspirin alone. Ann Intern med 2005. 142: 251-259. 